CrossMark \& click for updates

Cite this: J. Mater. Chem. B, 2016, 4,716

Received 20th October 2015, Accepted 14th December 2015

DOI: $10.1039 / c 5 t b 02191 j$

www.rsc.org/MaterialsB

\title{
Magnetic field responsive drug release from magnetoliposomes in biological fluids $\dagger$
}

\author{
Silvia Nappini, $\ddagger^{\mathrm{a}}$ Silvia Fogli, ${ }^{a}$ Benedetta Castroflorio, $\S^{\mathrm{a}}$ Massimo Bonini, ${ }^{\mathrm{a}}$ \\ Francesca Baldelli Bombelli ${ }^{b}$ and Piero Baglioni*a
}

\begin{abstract}
The final fate of nano-scaled drug delivery systems into the body is highly affected by their interaction with proteins in biological fluids (serum, plasma, etc.). Nanocarriers dispersed in biological fluids bear a protein "corona" that covers their surface. Thus, it is extremely important to evaluate the drug release efficiency also in the biological environment where protein-nanocarrier complexes are formed. The purpose of this work is to determine how drug release from lipid vesicle carriers is influenced by the interaction with serum proteins, highlighting the importance to test the effectiveness of such systems in the biological milieu. In particular, this paper describes the magnetically triggered release behaviour of magnetoliposomes (MLs) dispersed both in aqueous physiological buffer and in bovine serum at two different concentrations $(10 \%$ and $55 \% \mathrm{v} / \mathrm{v})$ upon exposure to a low-frequency alternating magnetic field (LF-AMF). We studied the release from MLs loaded with two types of magnetic nanoparticles (MNPs): citrate coated $\mathrm{Fe}_{3} \mathrm{O}_{4}$ and oleic acid coated $\gamma-\mathrm{Fe}_{2} \mathrm{O}_{3}$. The permeability in the above-mentioned fluids was evaluated in terms of the fluorescence self-quenching of carboxyfluorescein (CF) entrapped inside the liposome aqueous pool. The results showed a strong reduction of the release in biological fluids, in particular at high serum concentration. We related this decrease to the formation of protein-liposome complexes that, under LF-AMF exposure, are subjected to destabilization and tend to form aggregates. Our results clearly highlight the importance of testing the release efficiency of self-assembled drug delivery systems in biological fluids, in order to understand their behaviour in the presence of proteins and biomolecules.
\end{abstract}

\section{Introduction}

Lipid vesicles are now considered clinically established nanoscaled systems for the delivery of drugs, nucleic acids or agents for biomedical applications. ${ }^{1-3}$ Liposomes found large applicability because of their biocompatibility, flexibility in composition and size, ${ }^{4}$ as well as ability to encapsulate both hydrophilic and hydrophobic molecules into the aqueous pool $^{5,6}$ or within the lipid bilayer, ${ }^{7,8}$ respectively. One of the key features of a drug carrier is the release of the encapsulated drug selectively at

\footnotetext{
${ }^{a}$ Department of Chemistry "U. Schiff" and CSGI, via della Lastruccia 3, 50019 Sesto Fiorentino, Florence, Italy.E-mail: baglioni@csgi.unifi.it

${ }^{b}$ Centro Europeo di Nanomedicina (CEN), c/o Department of Chemistry, Materials and Chemical Engineering "Giulio Natta", Politecnico di Milano, Milano, Italy

$\dagger$ Electronic supplementary information (ESI) available: Description of the LF AMF setup, size distribution of MLs and liposomes in serum, optical microscopy images of MLs in serum, SAXS spectra of liposomes in serum, kinetics of release of liposomes, calibration curves of CF in serum, and the models for the fitting SAXS analysis. See DOI: 10.1039/c5tb02191j

¥ Present address: IOM-CNR, Laboratorio TASC, SS 14, Km 163,5, 34149 Basovizza, Trieste, Italy.

$\S$ Present address: Laboratory for Molecular Surfaces and Nanotechnology (LAMSUN), Department of Chemical Sciences and CSGI, Catania, Italy.
}

the target site with an efficient rate. ${ }^{9}$ In liposomes the diffusion of the drugs takes place spontaneously through the membrane, but the drug release rate could also be enhanced by an external stimulus, such as $\mathrm{pH},{ }^{10}$ temperature, ${ }^{11}$ and mechanical ablation (for example, using low frequency ultrasounds ${ }^{12,13}$ ). As we described previously, an efficient pathway towards the fast release of drugs from lipid vesicles is through the encapsulation of magnetic nanoparticles (MNPs) either in the membrane or inside the water pool. The release is then triggered by exposure to an alternating magnetic field (AMF). ${ }^{14,15}$ The magnetic NPs embedded in magnetoliposomes (MLs) can also be used to direct and accumulate the loaded drug to targeted sites by means of a magnetic gradient, ${ }^{14,16}$ eventually followed by the release of the drug by the application of the AMF., ${ }^{6,14,17,18}$

Most typically, a high-frequency alternating magnetic field (HF-AMF, 50-400 kHz) is used to promote local heating of magnetic NPs (hyperthermia), resulting in the thermal ablation of cells in their proximity, with limited damage to healthy tissues. ${ }^{11,14,19-22}$ Recently, it has been shown that the safer lowfrequency alternating magnetic field (LF-AMF, 0.01-10 kHz) can also be applied to promote drug release from magnetic nanocomposites. ${ }^{23-26}$ In our previous studies ${ }^{6,8,15}$ we studied the 
effect of the LF-AMF on ML permeability in the presence of both hydrophilic ${ }^{6}$ and hydrophobic ${ }^{8}$ cobalt ferrite $\left(\mathrm{CoFe}_{2} \mathrm{O}_{4}\right)$ NPs loaded in the aqueous pool and in the lipid bilayer, respectively.

Despite the promising results obtained with MLs in terms of controlled drug release, there are several barriers that have to be overcome before their effective use in vivo. The first aspect to take into account is their behaviour in biological fluids, where, it is now accepted, nano-surfaces are modified by the adsorption of biomolecules such as proteins and lipids forming a biomolecular protein corona. ${ }^{27}$ Many factors affect the formation of this corona, such as the surface curvature, size, chemical composition and protein concentration..$^{28,29}$ Similarly, proteins adsorb on the liposome membrane because of both hydrophobic and electrostatic interactions. ${ }^{30}$

It is thought that the protein corona determines the fate of the NPs in vivo, regulating the interactions with cells and causing their removal from the bloodstream. For example, the adsorption of opsonins like fibrinogen, IgG, and the complement factor is believed to promote phagocytosis with the removal of the NPs from the bloodstream, ${ }^{31}$ while binding of dysopsonins like human serum albumin (HSA), apolipoproteins, etc. promotes prolonged circulation time in blood. ${ }^{32}$ Furthermore, corona proteins can physically mask the NP surface, potentially affecting the therapeutic effect of molecules, antibodies, DNA oligomers, etc. bound to the NP surface. On the other hand, recent studies have shown that the presence of a protein corona could enhance the drug delivery action of NPs, promoting high payloads. Cifuentes-Rius et $a l .{ }^{33}$ have shown that the payload release profile of pre-formed NP-protein corona complexes (nanorods, gold nanobones, and carbon nanotubes) is strongly affected by different biological environments. ${ }^{33}$ In particular, fluids rich of hard corona proteins promoted a faster release of the payload than those bearing soft corona proteins.

Here, we report a detailed study about the behaviour of MLs dispersed in serum upon exposure to a LF-AMF in order to understand how the magnetically triggered release is influenced by the interaction with serum proteins. MLs were formed by 1-palmitoyl-2-oleoyl-sn-glycero-3-phosphocholine (POPC) embedded with two types of NPs: citrate-coated $\mathrm{Fe}_{3} \mathrm{O}_{4}$ NPS and oleic acid-coated $\gamma-\mathrm{Fe}_{2} \mathrm{O}_{3}$ NPs. A consistent number of reports (recently summarized in a comprehensive review ${ }^{34}$ ) have demonstrated that the citrate coating allows for the encapsulation of magnetic NPs within the liposome lumen, while the oleic acid coating takes to their embedding at the level of the lipid bilayer. MLs were dispersed in bovine serum at different protein concentrations $(10 \%$ and $55 \% \mathrm{v} / \mathrm{v}$ related to in vitro and in vivo protein concentrations in the biological environment) and the release was evaluated after exposure to a $5.7 \mathrm{kHz}$ alternating magnetic field. Control experiments were performed on the same MLs in aqueous physiological buffer (PBS) and on non-magnetic liposomes. As in previous reports, ${ }^{6,8,15}$ the enhancement of liposome permeability upon LF-AMF exposure was measured as the self-quenching decrease of the fluorescent molecule carboxyfluorescein (CF) entrapped in the liposome pool.

\section{Materials and methods}

\section{Materials}

Iron(III) chloride hexahydrate (97\%), iron(II) sulfate heptahydrate $(99 \%)$, iron pentacarbonyl $\mathrm{Fe}(\mathrm{CO})_{5}$, octyl ether (purity grade, 99\%), oleic acid (99\%), trimethylamine $N$-oxide (98\%), cyclohexane ( $>99.9 \%$ ), ethyl alcohol ( $>99.8 \%$ ), sodium hydroxide solution $33 \%$, hydrogen chloride solution 37\%, tetramethylammonium hydroxide $25 \%$ wt solution in water (TMAOH), sodium chloride $(>99.5 \%)$, tri-sodium citrate dihydrate $(>99 \%)$, citric acid monohydrate (>99.5\%), 5(6)-carboxyfluorescein (CF, > 95\% HPLC), chloroform (99.9\% HPLC grade), ethylenediaminetetraacetic acid (EDTA, 99.5\%), and Triton X-100 were purchased from Sigma-Aldrich. 1-Palmitoyl-2-oleoyl-sn-glycero-3-phosphocholine (POPC) was purchased from Avanti polar lipids. HEPES [4-(2hydroxyethyl) piperazine-1-ethane-sulfonic acid] (ultra »99.5\%) and concentrated nitric acid (90\%) were purchased from Fluka. Methanol (99.8\%) was purchased from Panreac Quimica Sau and Sephadex G-25 Superfine from Pharmacia Fine Chemicals. Fetal bovine serum was purchased from HyClone.

All the reagents were used as supplied, except for trimethylamine $N$-oxide that was dehydrated immediately before use.

\section{Synthesis of citrate coated $\mathrm{Fe}_{3} \mathrm{O}_{4}$ nanoparticles}

Magnetite $\left(\mathrm{Fe}_{3} \mathrm{O}_{4}\right)$ NPs were prepared by introducing minor modifications to a method described elsewhere. ${ }^{35}$ Briefly, a solution of $1 \mathrm{M} \mathrm{FeCl}_{3}(1 \mathrm{ml})$ in $2 \mathrm{M} \mathrm{HCl}$ was added to $4 \mathrm{ml}$ of $2 \mathrm{M} \mathrm{Fe}_{2} \mathrm{SO}_{4}$ solution in $2 \mathrm{M} \mathrm{HCl}$. An aqueous solution of $\mathrm{NH}_{3}$ was added dropwise to the mixture under vigorous agitation. A black precipitate of magnetite was formed immediately. The particles obtained were separated by magnetic decantation, washed with water, dispersed in $10 \mathrm{ml}$ of $2 \mathrm{M} \mathrm{HCl}$ solution and stirred for $5 \mathrm{~min}$. The precipitate was separated again by magnetic decantation and washed several times with water. The citrate coating of NPs was carried out according to a method reported in a previous study. ${ }^{6}$ The precipitate was dispersed in $20 \mathrm{ml}$ of $100 \mathrm{mM}$ citric acid solution and stirred for $1 \mathrm{~h}$ at room temperature. After recollecting the precipitate with the magnet, NPs were dispersed in $20 \mathrm{mM}$ trisodium citrate $(20 \mathrm{ml})$ and kept under stirring for $45 \mathrm{~min}$. The obtained particles were separated by magnetic decantation and washed several times with water and acetone in order to remove any excess of citric acid. The citrate coated NPs were gently dried under a nitrogen gas flux, dispersed in the buffer solution (10 mM PBS, $150 \mathrm{mM}$ $\mathrm{NaCl}, \mathrm{pH} 7.4$ ) and kept under stirring for $24 \mathrm{~h}$. Finally the dispersion was centrifuged at $1000 \mathrm{~g}$ for $2 \mathrm{~min}$ and the supernatant was dialyzed against water for $24 \mathrm{~h}$ through a cellulose dialysis bag (avg. flat width $23 \mathrm{~mm}$, MWCO 12400, 99.99\% retention) in order to remove non-adsorbed citrate species.

\section{Synthesis of oleic acid coated $\gamma-\mathrm{Fe}_{2} \mathrm{O}_{3}$ nanoparticles}

Maghemite $\left(\gamma-\mathrm{Fe}_{2} \mathrm{O}_{3}\right)$ NPs were prepared according to the method previously described by Hyeon et al. ${ }^{36}$ Oleic acid $(1.197 \mathrm{~g}, 6 \mathrm{mmol})$ was added to $15 \mathrm{ml}$ of octyl ether and the solution was heated up to $100{ }^{\circ} \mathrm{C}$. Iron pentacarbonyl $\mathrm{Fe}(\mathrm{CO})_{5}$ (260 $\mu \mathrm{l}, 2 \mathrm{mmol}$ ) was then added and the resulting mixture was 
refluxed for $1 \mathrm{~h}$, during which the colour of the solution changed from orange to brown-black. At the end of this step, the dispersion of NPs was cooled down to room temperature and purged with nitrogen. Still under a nitrogen atmosphere, dehydrated trimethylamine $\mathrm{N}$-oxide (447 $\mathrm{mg}, 6 \mathrm{mmol}$ ) was added and the dispersion was heated to $130{ }^{\circ} \mathrm{C}$ and kept at this temperature for $2 \mathrm{~h}$. The nitrogen flux was then removed and the temperature was slowly increased $\left(3{ }^{\circ} \mathrm{C} \mathrm{min}^{-1}\right)$ up to the boiling point. After refluxing for 2 hours, the dispersion was cooled to room temperature. NPs were then collected by adding ethanol $(50 \mathrm{ml})$ and the dispersion was centrifuged at $6000 \mathrm{rpm}$. The precipitate was washed twice with $20 \mathrm{ml}$ of ethanol and then the dried precipitate was finally dissolved in cyclohexane to obtain a stable magnetic fluid.

\section{Preparation of liposomes and magnetoliposomes}

The preparation of MLs in the presence of both hydrophilic and hydrophobic NPs has been previously described. ${ }^{6,8,15}$ The method is based on lipid film hydration followed by sequential extrusion. ${ }^{14,37,38}$ MLs loaded with citrate coated $\mathrm{Fe}_{3} \mathrm{O}_{4}$ NPs were prepared by the evaporation of the solvent from a $\mathrm{CHCl}_{3} / \mathrm{MeOH}$ solution of the lipid (POPC); the dry lipid film was hydrated with a buffer solution of carboxyfluorescein (30 mM CF, $10 \mathrm{mM}$ PBS, $130 \mathrm{mM} \mathrm{NaCl}, 94 \mathrm{mM} \mathrm{NaOH}, 1 \mathrm{mM}$ EDTA, pH 7.4) and $\mathrm{Fe}_{3} \mathrm{O}_{4}$ nanoparticles, so to have a lipid concentration of $20 \mathrm{mM}$.

MLs with hydrophobic $\gamma-\mathrm{Fe}_{2} \mathrm{O}_{3}$ NPs were prepared by evaporation of the solvent from a $\mathrm{CHCl}_{3} / \mathrm{MeOH}$ solution of the lipid and an aliquot of maghemite NPs coated with oleic acid in cyclohexane; the dry film was hydrated with the buffer solution of CF to have a lipid concentration of $20 \mathrm{mM}$. Finally, control liposomes (i.e. without magnetic NPs) were prepared by adding a buffered solution of CF to the dry lipid film.

The three dispersions were homogenized by vortex mixing and freeze-thaw six times. Multilamellar polydispersed vesicles were then sequentially extruded at room temperature through polycarbonate membranes (Whatman, $0.8 \mu \mathrm{m} / 0.4 \mu \mathrm{m} / 0.2 \mu \mathrm{m}$ pore size). Non-entrapped CF and NPs were removed by gel exclusion chromatography (GEC) using a Sephadex G-25 micro-column (1 ml syringe) saturated with a $20 \mathrm{mM}$ POPC solution. Liposome dispersions were eluted by centrifugation at $2000 \mathrm{~g}$ for 3 minutes without the loss or dilution of the material. The collected fractions were investigated by DLS and checked for their NP content by ICP-AES.

\section{Inductively coupled plasma-atomic emission spectrometry (ICP-OES)}

A VARIAN 720 OES inductively coupled plasma optical emission spectrometer (ICP-OES) was used for the determination of $\mathrm{Fe}$ content (ppm) in both magnetite and maghemite fluids, and inside MLs. The samples were diluted from $0.1 \mathrm{ml}$ to $5.0 \mathrm{ml}$ in $0.1 \%$ nitric acid. A calibration curve of standard solutions of $\mathrm{Fe}$ was used (Fe 238.204; 259.940; $234.350 \mathrm{~nm}$ ). An internal standard (Ge, 5 ppm, $209.426 \mathrm{~nm}$ ) was used to correct for matrix effects.

\section{Magnetic field generator}

A sinusoidal adjustable magnetic field was generated in the gap of a broken ferrite ring carrying a solenoid through which an alternating electric current (AC) from a tone generator was led. Samples to be treated with the LF-AMF were placed in the middle of the gap within $1 \mathrm{~cm}$ cylindrical quartz cells. Due to the design of the experimental apparatus, the magnetic field inside the cell is not isotropic. As a consequence, the sample undergoes magnetic field gradients that cannot be avoided (see ESI, $\uparrow$ Fig. S1 and S2). The temperature of the solutions treated with the LF-AMF was checked immediately after each treatment, showing that no bulk hyperthermic effect was generated.

\section{Quasi-elastic light scattering (QELS)}

QELS measurements were carried out by means of a 90Plus/ BI-MAS system using a Brookhaven Instrument apparatus (New York, USA). The light source was a $15 \mathrm{~mW}$ solid state laser $(\lambda=635 \mathrm{~nm})$. Measurements were performed at $25{ }^{\circ} \mathrm{C}$. Data analysis has been performed according to standard procedures, and interpreted through a Cumulant expansion of the field autocorrelation function, arrested to the second order. Moreover, in order to obtain a distribution $w(\Gamma)$ of decay rates, a non-negatively constrained least squares (NNLS) ${ }^{39}$ algorithm was used to invert the experimental data.

From the decay rates, $\Gamma$, through the equation $\Gamma=D_{\mathrm{t}} \cdot q^{2}$ which is valid for diffusive modes, we can determine the translational diffusion coefficient, $D_{\mathrm{t}}$. The diffusion coefficients provide access to the hydrodynamic correlation lengths $R_{\mathrm{H}}$ for isotropic particles through the Stokes-Einstein relationship

$$
D_{\mathrm{t}}=k_{\mathrm{B}} T / 6 \pi \eta_{\mathrm{S}} R_{\mathrm{H}}
$$

where $\eta_{\mathrm{S}}$ is the solvent viscosity and $k_{\mathrm{B}}$ is the Boltzmann constant. The particle size distributions resulting from QELS are provided as scattered intensity $v s$. hydrodynamic diameter to account for the higher sensitivity of the technique towards bigger scattering objects.

\section{Zeta potential}

Zeta potential analyses were performed using a ZetaPlus system by means of a 90Plus/BI-MAS system using a Brookhaven Instrument apparatus (New York, USA). A laser beam (35 mW solid state laser, $\lambda=635 \mathrm{~nm}$ ) passes through an optical cell that carries two electrodes to generate the electric field. Measurements of liposomes and magnetoliposomes dispersed in water were performed at $25{ }^{\circ} \mathrm{C}$ and analyzed by the Smoluchowski approximation.

\section{Small angle X-ray scattering (SAXS)}

SAXS measurements were carried out using a HECUS SWAXcamera (Kratky) equipped with a position-sensitive detector (OED $50 \mathrm{M}$ ) containing 1024 channels of $54 \mu \mathrm{m}$ width. An $\mathrm{X}$-ray generator (Seifert ID-3003), operating at a maximum power of $2 \mathrm{~kW}$, provides $\mathrm{Cu} \mathrm{K}_{\alpha}$ radiation of wavelength $1.542 \AA$ A. A $10 \mu \mathrm{m}$ thick nickel filter was used to remove the $\mathrm{Cu} \mathrm{K}_{\beta}$ radiation. The volume between the sample and the detector was kept under vacuum $(P<1 \mathrm{mBar})$ during measurements to minimize scattering from air. Samples were filled into $1 \mathrm{~mm}$ quartz capillaries. Measurements were carried out at $25{ }^{\circ} \mathrm{C}$ and temperature was controlled using a Peltier element, with an 
accuracy of $\pm 0.1{ }^{\circ} \mathrm{C}$. All scattering curves were corrected for solvent and empty cell contribution. The data were slit desmeared by a linear method. ${ }^{40}$

In SAXS experiments, the scattered radiation intensity, $I(Q)$, is given by: ${ }^{41}$

$$
I(Q)=A \cdot \Phi \cdot P(Q) \cdot S(Q)+I_{\mathrm{bkg}}
$$

where $A$ is the amplitude accounting for the instrumental factor, $\Phi$ is the volume fraction, $P(Q)$ is the intra-particle structure factor, $S(Q)$ is the inter-particle structural factor accounting for the mutual particle correlations, $I_{\mathrm{bkg}}$ is the incoherent background and $Q$ is the scattering vector.

The scattering length densities (SLDs) of magnetic NPs, liposomes and MLs were calculated from the X-ray scattering lengths of the atoms, considering the molecular volumes of phospholipid fragments evaluated by Armen et al. ${ }^{42}$ The SLD of each component was kept constant during the fitting of the experimental data. ${ }^{43}$

\section{Drug release experiments}

Steady-state fluorescence was measured using a LS50B spectrofluorimeter (Perkin Elmer, Italy). The emission fluorescence spectra of CF were recorded between 500 and $610 \mathrm{~nm}$ in the corrected spectrum mode with the excitation wavelength set at $492 \mathrm{~nm}$ (slit $2.5 \mathrm{~nm}$ ). At least 5 scans were averaged for each spectrum. The release behaviour of MLs and liposomes (diluted 1:67 with PBS and bovine serum $10 \%$ and $55 \% \mathrm{v} / \mathrm{v}$ ) was followed as a function of the LF-AMF exposure time by measuring the fluorescence intensity every $10 \mathrm{~min}$ for 15 hours. Fluorescence experiments were also performed on MLs and liposomes maintained at the highest temperature reached during the magnetic treatment, for the same exposure time. The mother solution of all samples was diluted to the measurement concentration with a solution of Triton X-100 to achieve complete release of CF through vesicle disruption ( $\left.I_{\mathrm{MAX}}\right)$. The release percentage was calculated from the fluorescence intensity as:

$$
\%_{\text {release }}=100 \cdot\left[I_{\mathrm{ML}}(t)-I_{\mathrm{ML}}(0)\right] /\left[I_{\mathrm{MAX}}-I_{\mathrm{ML}}(0)\right]
$$

where $I_{\mathrm{ML}}(t)$ is the measured fluorescence intensity, $I_{\mathrm{ML}}(0)$ is the fluorescence intensity of the untreated sample, and $I_{\mathrm{Max}}$ is the maximum fluorescence emission after Triton X-100 addition. However, the interaction of Triton X-100 with serum makes the direct measurements of $I_{\text {MAX }}$ in biological medium very difficult; for this reason the theoretical value of $I_{\mathrm{MAX}}$ for MLs dispersed in biological fluids was extrapolated from the calibration curves of CF in serum $10 \%$ and $55 \% \mathrm{v} / \mathrm{v}$ using the experimental value of $I_{\text {MAX }}$ obtained for MLs in PBS as reference (see ESI, $\dagger$ Fig. S12).

\section{Results and discussion}

\section{Characterization of the magnetic nanoparticles}

SAXS spectra of both citrate coated $\mathrm{Fe}_{3} \mathrm{O}_{4}$ and oleic acid coated $\gamma-\mathrm{Fe}_{2} \mathrm{O}_{3}$ NPs are reported in Fig. 1 together with the best fitting curves. $\mathrm{Fe}_{3} \mathrm{O}_{4}$ NPs dispersed in PBS were modelled according to the formalism introduced by Bartlett and Ottewill for

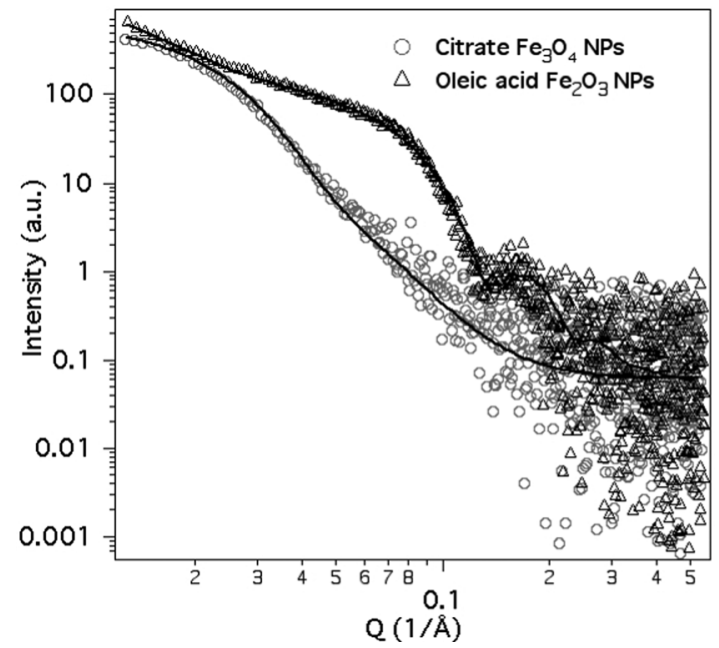

Fig. 1 SAXS spectra of citrate coated $\mathrm{Fe}_{3} \mathrm{O}_{4}$ in PBS $(O)$ and oleic acid coated $\gamma-\mathrm{Fe}_{2} \mathrm{O}_{3}(\triangle)$ NPs in cyclohexane.

Table 1 Structural features of the magnetic NPs extracted from the SAXS measurements

\begin{tabular}{llc}
\hline & Citrate $\mathrm{Fe}_{3} \mathrm{O}_{4}$ & Oleic acid $\gamma-\mathrm{Fe}_{2} \mathrm{O}_{3}$ \\
\hline SAXS fitting & Schulz sphere & Pearl necklace model \\
$\langle R\rangle[\mathrm{nm}]$ & $7.7 \pm 0.1$ & $3.3 \pm 0.3$ \\
Shell [nm] & - & $1.2 \pm 0.3$ \\
Polydispersity & $0.3 \pm 0.01$ & $0.10 \pm 0.02$ \\
Fractal dimension, $D$ & - & $2.8 \pm 0.1$ \\
NP correlation length, $\xi[\mathrm{nm}]$ & - & $31.1 \pm 0.3$
\end{tabular}

polydispersed spherical particles. ${ }^{44}$ In this approach, the particles are described as spherical objects with a uniform scattering length density and a Schulz distribution of radii. ${ }^{45,46}$

No structure factors were included in this model as, due to the low concentration of particles in the dispersions, the interparticle scattering effects are negligible. The fitting returned NPs of about $7.7 \mathrm{~nm}$ in radius.

SAXS spectra of $\gamma-\mathrm{Fe}_{2} \mathrm{O}_{3}$ NPs dispersed in cyclohexane were analysed according to the "pearl necklace" model, ${ }^{47}$ described in detail in the ESI. $\dagger$ This model was developed to describe the scattering pattern produced by fractal assemblies of micelles (i.e. spherical objects) templated by a backbone. ${ }^{41}$ The structural parameters extracted by the fitting are reported in Table 1. The best fitting was obtained for NPs having a radius of about $4.5 \mathrm{~nm}$ (a core of $3.3 \mathrm{~nm}$ and a shell of $1.2 \mathrm{~nm}$ ). The resulting fractal dimension (2.8) clearly indicates that $\gamma-\mathrm{Fe}_{2} \mathrm{O}_{3}$ NPs were arranged into rather compact clusters. Moreover, the correlation length value of about $31 \mathrm{~nm}$ indicates that NP aggregates were composed of units formed by few NPs. It should be stressed that the correlation length value should be taken cautiously and it could represent an under-estimation, as its value approaches the instrumental limits of our SAXS.

\section{Characterization of magnetoliposomes}

MLs and control liposomes were characterized in PBS, 10\% serum and $55 \%$ serum by DLS and SAXS measurements. In Table 2 the main features of the investigated MLs are reported. 
The loading efficiency of CF and MNPs inside MLs was determined by measuring their concentration before and after gel exclusion chromatography (GEC) purification.

CF concentration inside MLs was extrapolated from the fluorescence intensity of the dye after vesicle disruption by adding Triton $\mathrm{X}-100$. This value corresponds also to the maximum fluorescence emission $\left(I_{\max }\right)$ of the sample when CF is completely released. The loading efficiency (estimated between 4 and $15 \%$ depending on the sample) was calculated as the ratio of $\mathrm{CF}$ concentration before (known value) and after GEC purification.

NPs' concentration before and after GEC purification was determined by ICP-OES, and the loading efficiency was calculated as their ratio (see Table 2).

The average hydrodynamic diameters, $D_{\mathrm{H}}$, of the liposomes in PBS were obtained by fitting the autocorrelation functions using cumulant analysis. The size distributions obtained by NNLS analysis are reported in Fig. 2(a). Empty liposomes and MLs containing citrate coated $\mathrm{Fe}_{3} \mathrm{O}_{4}$ NPs resulted in a single population (monomodal distribution) centred nearly at the same $D_{\mathrm{H}}$ value obtained by the cumulant analysis, MLs containing oleic acid $\gamma-\mathrm{Fe}_{2} \mathrm{O}_{3}$ NPs showed instead the presence of two populations. The smaller size distribution is consistent with the presence of the NP clusters found by SAXS, eventually surrounded by a phospholipid layer to make them stable in a hydrophilic environment. The other distribution consists of objects larger than reference liposomes, which could be the result of a partial aggregation driven by the presence of NPs. DLS analysis of the same samples was also performed immediately after $15 \mathrm{~min}$ of exposure to the AMF at a frequency of $5.7 \mathrm{kHz}$. This frequency was chosen on the base of previous results. ${ }^{6,8,15}$ DLS analysis of magnetic-treated samples did not show large variations in the ML size distribution, except for those loaded with oleic acid-coated NPs, as shown in Fig. 2(b). The presence of hydrophobic NPs into the liposome bilayer promotes therefore the aggregation between vesicles during the AMF exposure, more likely due to local MNP motions and hyperthermic effects: in fact, previous ACsusceptibility measurements performed on MLs embedded with $\mathrm{CoFe}_{2} \mathrm{O}_{4}$ NPs showed that, for field frequency higher than $10 \mathrm{~Hz}$, the local hyperthermia is effective. ${ }^{6}$

DLS analyses were also performed on samples dispersed in serum at a protein concentration of $10 \%$ and $55 \% \mathrm{v} / \mathrm{v}$, and the results are reported in Fig. S3 in the ESI. $\dagger$ However, it was very difficult to evaluate the size distribution of protein-liposome complexes in serum through DLS measurements due to the

Table 2 MNP loading in magnetoliposomes dispersed in PBS

\begin{tabular}{llllll}
\hline $\mathrm{NPs}^{a}$ & $\begin{array}{l}{[\mathrm{NPs}]^{b}} \\
\left(\mathrm{mg} \mathrm{L}{ }^{-1}\right)\end{array}$ & $\begin{array}{l}\langle R\rangle^{c} \\
(\mathrm{~nm})\end{array}$ & $\begin{array}{l}\left\langle D_{\mathrm{H}}\right\rangle^{d} \\
(\mathrm{~nm})\end{array}$ & $\begin{array}{l}z \text {-pot. } \\
(\mathrm{mV})\end{array}$ & $\begin{array}{l}\text { NP loading } \\
\text { efficiency }^{f}(\%)\end{array}$ \\
\hline $\mathrm{Fe}_{3} \mathrm{O}_{4}$ citrate & 62 & 7.7 & 178.4 & -16.36 & 52 \\
$\gamma-\mathrm{Fe}_{2} \mathrm{O}_{3}$ oleic acid & 4.78 & 4.5 & 116.8 & -0.45 & 31
\end{tabular}

${ }^{a}$ Types of magnetic nanoparticles (MNPs). ${ }^{b}$ Magnetic NP concentration by ICP-OES after GEC purification. ${ }^{c}$ Average radius of nanoparticles by SAXS analysis. ${ }^{d}$ Average hydrodynamic diameter of MLS from DLS analysis. ${ }^{e}$ Surface charge of MLs from $z$-potential analysis.

${ }^{f}$ Calculated as $\left\{[\mathrm{NPs}]_{\text {after GEC }} /[\mathrm{NPs}]_{\text {before GEC }}\right\} \times 100$.
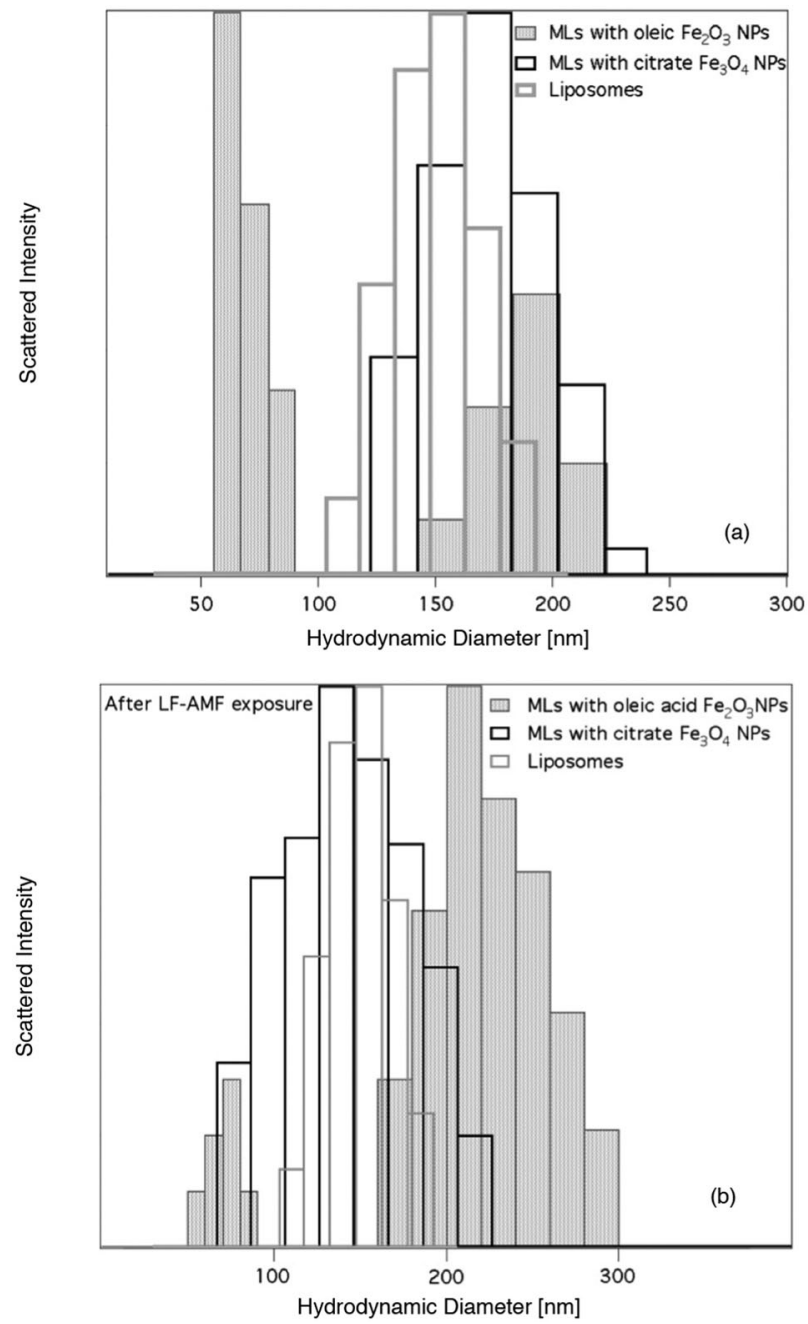

Fig. 2 Representative size distributions of liposomes (empty grey bars), magnetoliposomes with citrate coated $\mathrm{Fe}_{3} \mathrm{O}_{4}$ NPs (empty black bars) and magnetoliposomes with oleic acid coated $\mathrm{Fe}_{2} \mathrm{O}_{3} \mathrm{NPs}$ (grey bars) dispersed in PBS from NNL analysis before LF-AMF exposure (a) and after magnetic treatment at $5.7 \mathrm{kHz}$ for $15 \mathrm{~min}$ (b).

high background signal from the free proteins as reported in Fig. S4 (ESI $\dagger$ ). It was possible to detect the presence of a main population (not found in pure serum) of objects with a hydrodynamic size of about $500 \mathrm{~nm}$ in serum $10 \%$, and of about $700 \mathrm{~nm}$ in serum 55\% (see Fig. S3 in the ESI $\dagger$ ). The same behaviour was observed with both MLs loaded with citrate and oleic acid coated NPs. Thus, while no definitive conclusions could be extracted from the DLS analysis in serum, the results highlighted the formation of aggregates of MLs in the presence of the proteins and the synergistic effect of the AMF over aggregation (see Fig. S5 in the ESI $\dagger$ ). The aggregation of both empty liposomes and MLs in biological fluids after the magnetic treatment was also investigated by optical microscopy (see Fig. S6-S9 in the ESI $\dagger$ ). Phase-contrast micrographs showed large aggregates ranging from 0.2 to $3 \mu \mathrm{m}$ for liposomes and from 1 to $10 \mu \mathrm{m}$ for MLs. These results further confirmed the effect of the AMF on promoting the formation of large proteinvesicle agglomerates. 
SAXS measurements were carried out to obtain insights into the local arrangement of the lipid membrane as a function of NP encapsulation, AFM exposure and incubation in serum.

SAXS spectra of liposomes and MLs loaded with citratecoated NPs and oleic acid-coated NPs dispersed in PBS are reported in Fig. 3, together with the corresponding best fittings. SAXS spectra of liposomes were analyzed according to a model proposed by Nallet et $a{ }^{48}{ }^{48}$ for lamellar phases of the amphiphilic bilayer. Magnetoliposomes were modelled by taking into account the scattering intensity arising from both liposomes (amphiphilic bilayer) and NPs arranged in fractal clusters. Magnetic NPs were modelled according to the pearl necklace model, ${ }^{41}$ where each spherical NP has a constant shell thickness and a core with a Schulz distribution of radii. ${ }^{49}$ More details about the models and the fitting parameters are given in the ESI. $\dagger$ The results from the fitting (Table 3) show that citrate coated $\mathrm{Fe}_{3} \mathrm{O}_{4}$ NPs did not significantly affect the structure of the lipid bilayer, confirming their confinement within the aqueous pool of the liposomes. On the other hand, the change of both the lipid tail length and the headgroup thickness in the presence of oleic acid coated $\gamma-\mathrm{Fe}_{2} \mathrm{O}_{3}$ NPs clearly indicates the direct interaction of NPs with the lipid bilayer.

This could correspond to either a physisorption of MNPs onto the liposome or an insertion of the MNPs into the lipid bilayer. In fact, given that $\gamma-\mathrm{Fe}_{2} \mathrm{O}_{3}$ NPs diameters exceed the thickness of the lipid bilayer, the POPC membrane can distort to accommodate NPs both inside and/or on the surface of the lipid membrane. This result is consistent with the ability of the cell membrane to accommodate transmembrane proteins. ${ }^{50}$

SAXS spectra of liposomes in serum were also recorded (see Fig. S10 in the ESI $\dagger$ ), but the scattered intensity from serum does not allow for a reliable analysis of the results in terms of the effect of MNPs on the liposome bilayer.

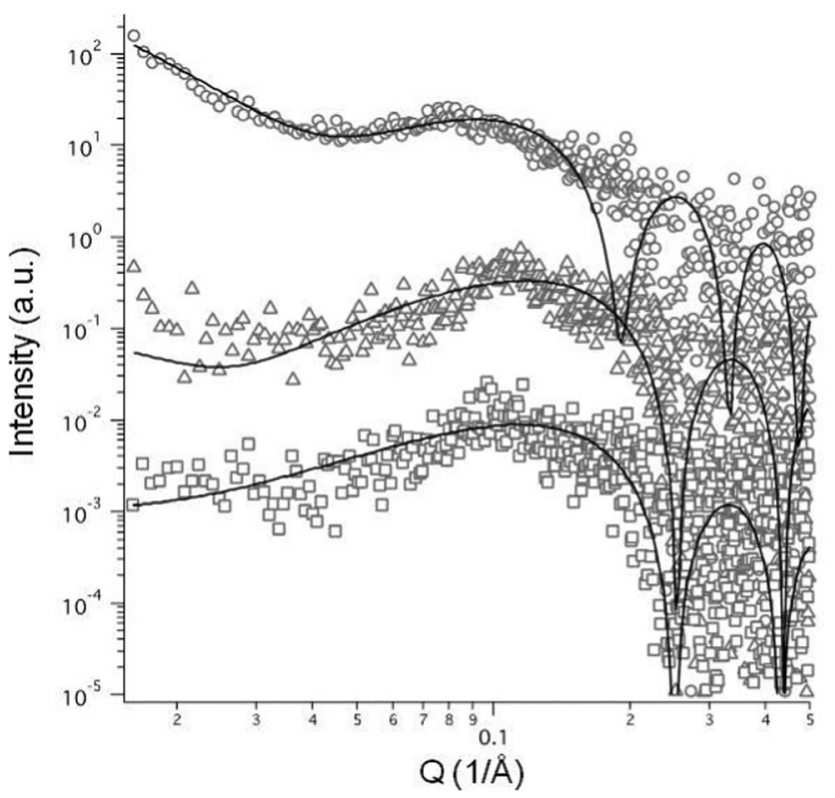

Fig. 3 SAXS spectra of liposomes ( $\square$ ), magnetoliposomes with citrate coated $\mathrm{Fe}_{3} \mathrm{O}_{4}(\triangle)$ and oleic acid coated $\gamma-\mathrm{Fe}_{2} \mathrm{O}_{3}(O)$ nanoparticles.
Table 3 Structural parameters of liposomes and MLs by SAXS analysis

\begin{tabular}{llll}
\hline & Liposomes & $\begin{array}{l}\text { MLs with } \\
\text { citrate } \mathrm{Fe}_{3} \mathrm{O}_{4}\end{array}$ & $\begin{array}{l}\mathrm{MLs} \text { with oleic } \\
\text { acid } \gamma-\mathrm{Fe}_{2} \mathrm{O}_{3}\end{array}$ \\
\hline $\begin{array}{l}\text { Lipid head group thickness, } \\
\delta_{\mathrm{H}}[\mathrm{nm}]\end{array}$ & 0.32 & 0.33 & 0.69 \\
Lipid tail length, $\delta_{\mathrm{T}}[\mathrm{nm}]$ & 1.63 & 1.61 & 2.27 \\
NP mean core radius, & - & 7.7 & 3.3 \\
$\langle R\rangle[\mathrm{nm}]$ & & & \\
NP shell thickness, $t[\mathrm{~nm}]$ & - & 0 & 1.2 \\
NP core polydispersity, $\sigma_{\mathrm{e}}$ & - & 0.3 & 0.15 \\
NP fractal dimension, $D$ & - & 2.98 & 2.8 \\
NP correlation length, & - & 60 & 11.8 \\
$\xi[\mathrm{nm}]$ & & &
\end{tabular}

\section{Release studies with carboxyfluorescein}

CF fluorescence was measured over time to investigate the release properties of untreated samples, and samples exposed to the LF-AMF for $15 \mathrm{~min}$. Fluorescence emission was related to $\mathrm{CF}$ release and was continuously monitored during the first 1000 minutes (about $16 \mathrm{~h}$ ) every $10 \mathrm{~min}$ and eventually, single measurements were performed 18, 24 and $40 \mathrm{~h}$ after the exposure. Release experiments were performed on the samples dispersed in three different investigated fluids (PBS, 10\% v/v serum and $55 \% \mathrm{v} / \mathrm{v}$ serum). The experimental data were fitted using the Ritger-Peppas equation, ${ }^{51,52}$ a semi-empirical equation used to describe drug release from polymeric systems:

$$
M_{t} / M_{\infty}=K \cdot t^{n} \text { for } M_{t} / M_{\infty}<0.6
$$

where $M_{t} / M_{\propto}$ is the drug fraction released at time $t, K$ is a kinetic constant that includes the structural and geometric characteristics of the system, and $n$ is the diffusion exponent indicative of the drug transport mechanism. The power-law equation can be seen as the superposition of a Fickian diffusion and a zero order kinetics: ${ }^{51,53} n$ values at around 0.5 indicate pure Fickian diffusion, while $n$ values at around 1.0 indicate that erosion or relaxation processes lead the release process (zero-order release, case II transport). Intermediate $n$ values between 0.5 and 1.0 suggest that both diffusion and erosion contribute to the overall release mechanism (anomalous transport). Occasionally, values of $n \gg 1$ have been observed and considered as kinetics dominated by disruption processes of the matrix (supercase II transport).

The kinetics of CF release from MLs containing oleic acidcoated $\gamma-\mathrm{Fe}_{2} \mathrm{O}_{3}$ NPs and citrate-coated $\mathrm{Fe}_{3} \mathrm{O}_{4}$ NPs are shown in Fig. 4 and 5, respectively, while those from pristine liposomes are reported in the $\mathrm{ESI} \dagger$ (Fig. S11). The kinetic parameters obtained from fitting according to eqn (4) are shown in Table 4. All the samples, including pristine liposomes, showed a lower release in serum than in PBS (see Fig. 4 and 5), indicating a trend typical of anomalous transport and zero-order kinetics of perturbed systems, most likely due to the formation of proteinvesicle adducts.

Focusing on the release profiles of MLs loaded with oleic acid $\gamma-\mathrm{Fe}_{2} \mathrm{O}_{3}$ NPs, two important aspects should be highlighted. First of all, the release profiles in buffer and serum 10\% when no LF-AMF is applied are very similar. The increase in serum proteins (serum 55\%) significantly affects both the profile and 

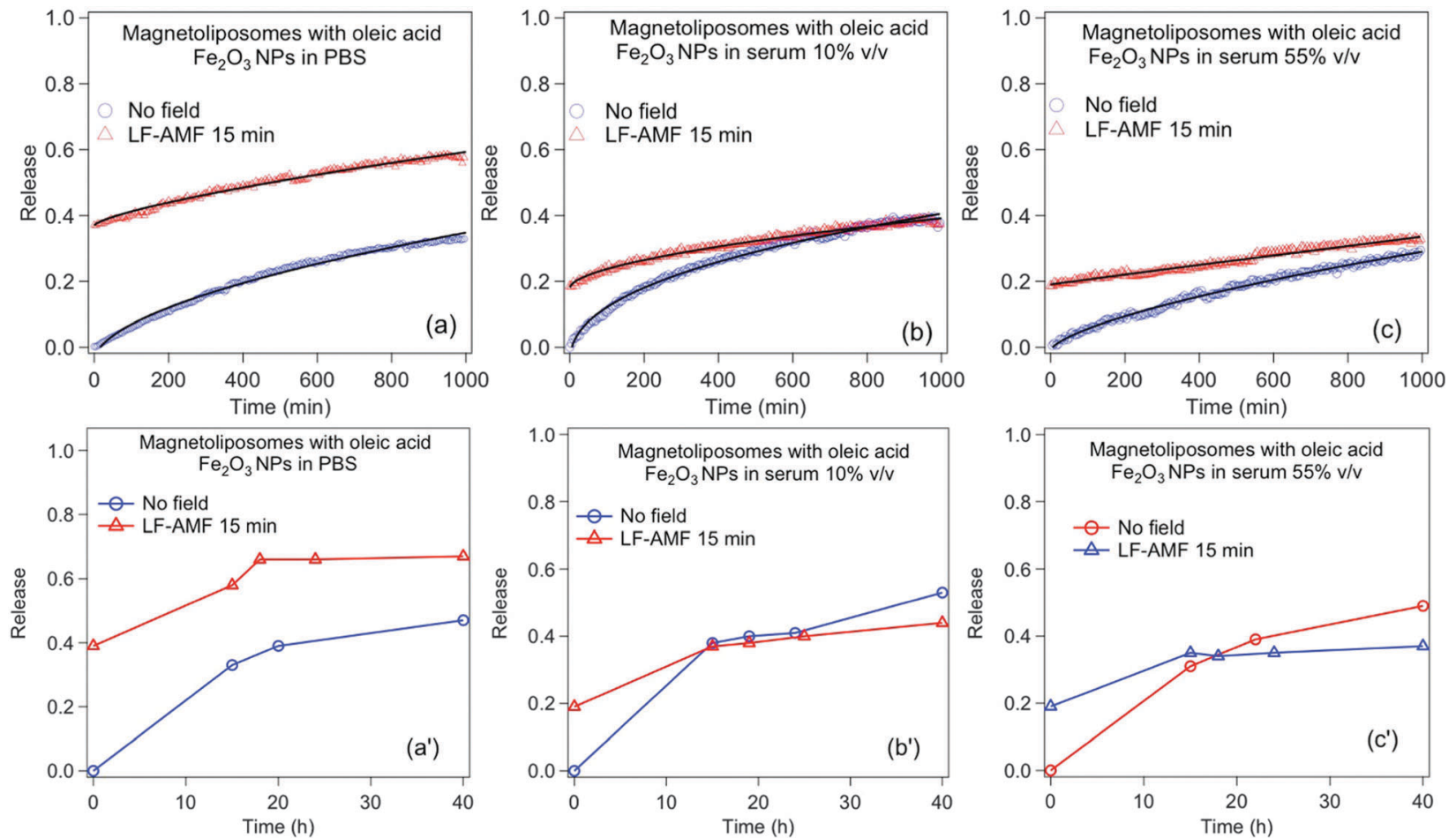

Fig. 4 Release kinetics of oleic acid-coated $\gamma$ - $\mathrm{Fe}_{2} \mathrm{O}_{3}$ NP embedded-magnetoliposomes for the first $16 \mathrm{~h}$. (O) Untreated samples, and ( $\triangle$ ) samples exposed to the AMF for 15 minutes at a frequency of $5.7 \mathrm{kHz}$ in PBS (a), serum $10 \% \mathrm{v} / \mathrm{v}$ (b), and serum $55 \% \mathrm{v} / \mathrm{v}$ (c). Solid curves are the best fitting obtained by means of eqn (4). Single measurements of the release in PBS $\left(a^{\prime}\right)$, serum $10 \% v / v\left(b^{\prime}\right)$, and serum $55 \%$ v/v $\left(c^{\prime}\right)$ were taken for 40 hours.
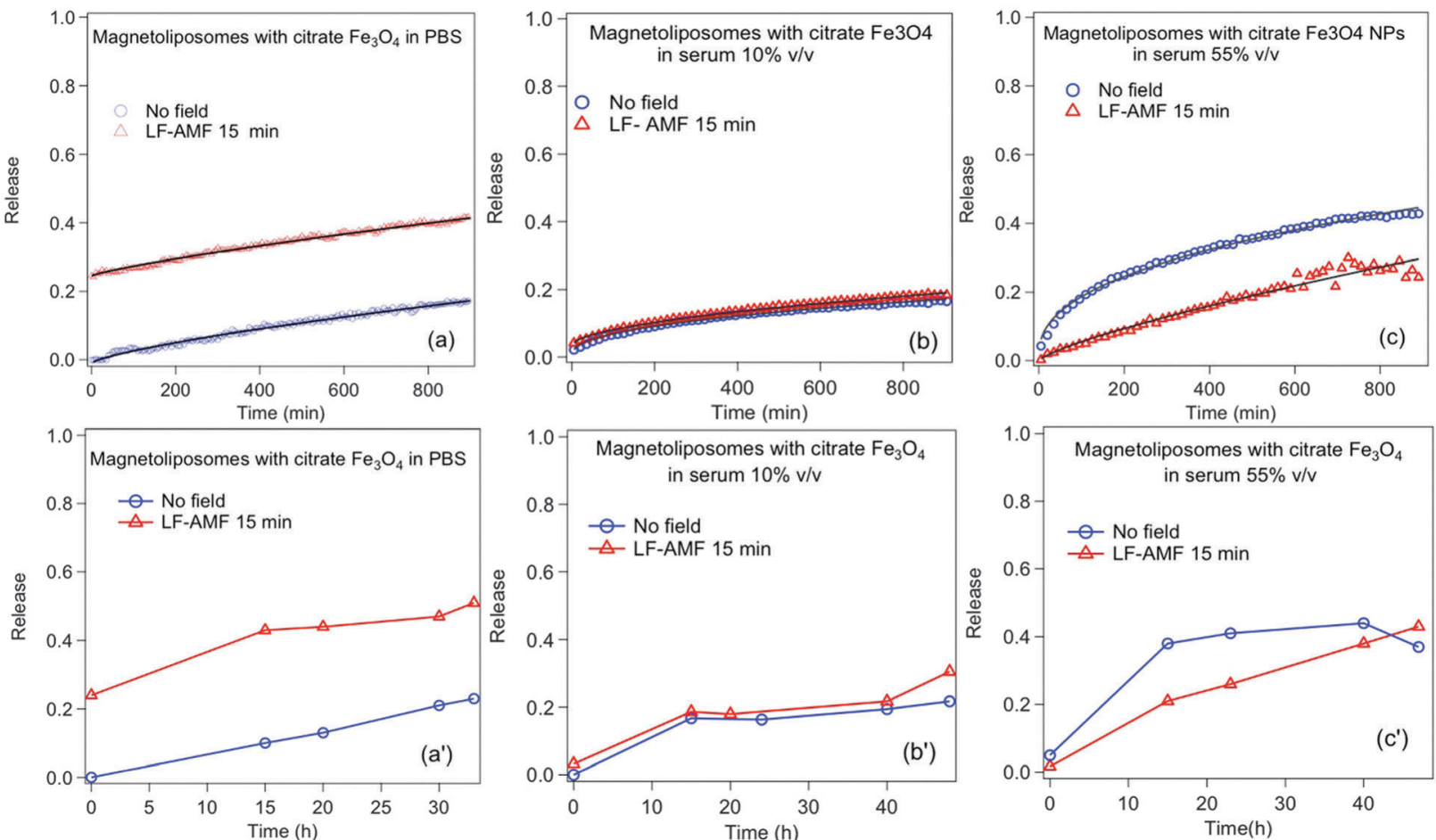

Fig. 5 Release kinetics of citrate-coated $\mathrm{Fe}_{3} \mathrm{O}_{4} \mathrm{NP}$ embedded-magnetoliposomes for the first $16 \mathrm{~h}$. ( $O$ ) Untreated samples, and $(\triangle)$ samples exposed to the AMF for 15 minutes at a frequency of $5.7 \mathrm{kHz}$ in PBS (a), serum $10 \% \mathrm{v} / \mathrm{v}(\mathrm{b})$, and serum $55 \% \mathrm{v} / \mathrm{v}$ (c). Solid curves are the best fitting obtained by means of eqn (4). Single measurements of the release in PBS $\left(a^{\prime}\right)$, serum $10 \% \mathrm{v} / \mathrm{v}\left(b^{\prime}\right)$, and serum $55 \% \mathrm{v} / \mathrm{v}\left(c^{\prime}\right)$ were taken within 40 hours. 
Table 4 Kinetic parameters obtained by fitting CF release curves from magnetoliposomes according to eqn (4)

\begin{tabular}{|c|c|c|c|}
\hline & & MLs with oleic acid $\gamma-\mathrm{Fe}_{2} \mathrm{O}_{3}$ NPs & MLs with citrate $\mathrm{Fe}_{3} \mathrm{O}_{4}$ NPs \\
\hline No field & $n$ & $0.55 \pm 0.01$ & $0.71 \pm 0.01$ \\
\hline & $K$ & $8.67 \times 10^{-3} \pm 0.88 \times 10^{-3}$ & $1.16 \times 10^{-3} \pm 0.07 \times 10^{-3}$ \\
\hline & $K$ & $4.66 \times 10^{-4} \pm 0.26 \times 10^{-4}$ & $7.21 \times 10^{-4} \pm 0.37 \times 10^{-4}$ \\
\hline No field & SERUM $10 \% \mathrm{v} / \mathrm{v}$ & & \\
\hline \multirow{2}{*}{ LF-AFM } & $n$ & $0.63 \pm 0.01$ & $0.68 \pm 0.01$ \\
\hline & $K$ & $1.70 \times 10^{-5} \pm 0.16 \times 10^{-3}$ & $1.06 \times 10^{-3} \pm 0.01 \times 10^{-3}$ \\
\hline \multirow{3}{*}{ No field } & SERUM 55\% v/v & & \\
\hline & $n$ & $0.66 \pm 0.01$ & $0.41 \pm 0.01$ \\
\hline & K & $3.13 \times 10^{-3} \pm 0.03 \times 10^{-3}$ & $2.65 \times 10^{-2} \pm 0.10 \times 10^{-2}$ \\
\hline LF-AFM & $n$ & $0.99 \pm 0.01$ & $0.77 \pm 0.03$ \\
\hline
\end{tabular}

the magnitude of the release profile, clearly suggesting a decrease in the permeability of the liposome membrane in the presence of a higher concentration of serum proteins (see Fig. 4a-c). Analysing the results in magnetically treated MLs loaded with oleic acid $\gamma-\mathrm{Fe}_{2} \mathrm{O}_{3} \mathrm{NPs}$, it is clear that the presence of serum proteins strongly mitigates the effect of the LF-AMF. Nevertheless, in all the samples the application of the LF-AMF induces a significant increase in the released amount of CF during its application and in the following few hours. In both $10 \%$ and $55 \%$ serum samples, after nearly 15 hours the release is comparable to that of untreated MLs.

This suggests that the initial release was promoted by the application of the magnetic field, most likely thanks to the defects generated at the bilayer level by the motions and the local heating of MNPs. Once the field was switched off, the release rate decreased, reasonably because of a healing effect favoured by the formation of protein-ML complexes. At very long times (i.e., after 40 hours) the behaviour is inverted, with magnetically treated MLs showing a released amount lower than the corresponding untreated samples. This behaviour further suggests an effect of the MNPs at the membrane level and a stabilization of the bilayer due to serum. The release from MLs loaded with citratecoated $\mathrm{Fe}_{3} \mathrm{O}_{4}$ NPs is even more affected by the presence of serum (see Fig. 5a-c). The effect of the LF-AMF on the released amount in PBS is very similar to what was observed with $\gamma-\mathrm{Fe}_{2} \mathrm{O}_{3}$ NPs, with a magnitude of the release nearly halved, even though the profiles are similar. When serum is present at $10 \%$, the release from untreated MLs is nearly identical to that in PBS, while increasing the amount of serum at 55\% brings a significant increase in the magnitude of release even without the magnetic field. This can be explained by the aggregation of MLs in the presence of a consistent amount of serum proteins and the consequent destabilization of the bilayer. This behaviour was not observed with $\gamma-\mathrm{Fe}_{2} \mathrm{O}_{3}$ NPs, most likely because of the direct interaction of the hydrophobic particles with the bilayer.

When serum is present at $10 \%$, the results show no effect of the LF-AMF on citrate-coated $\mathrm{Fe}_{3} \mathrm{O}_{4}$ NPs, while the sample with serum at 55\% shows a lower release when the LF-AMF is applied, converging at long times to the same value found when no
LF-AMF is applied. These results demonstrate that the application of the magnetic field in the presence of both citrate-coated $\mathrm{Fe}_{3} \mathrm{O}_{4}$ NPs and serum proteins induces a significant decrease in the membrane permeability thanks to the magnetically induced exposition of the particles towards the continuous phase surrounding the MLs. The surface reactivity of $\mathrm{Fe}_{3} \mathrm{O}_{4}$ NPs (citrate is weakly coordinated to their surface) makes them very likely to get coated by serum proteins already at the bilayer level, eventually making it less permeable.

The low response of MLs loaded with citrate-coated $\mathrm{Fe}_{3} \mathrm{O}_{4}$ NPs dispersed in serum can be also associated with the higher concentration of hydrophilic NPs that can be encapsulated in MLs in comparison to the hydrophobic ones. It is clear that a larger number of NPs promote serum protein adsorption on the ML membrane, thus the formation of larger serum-ML

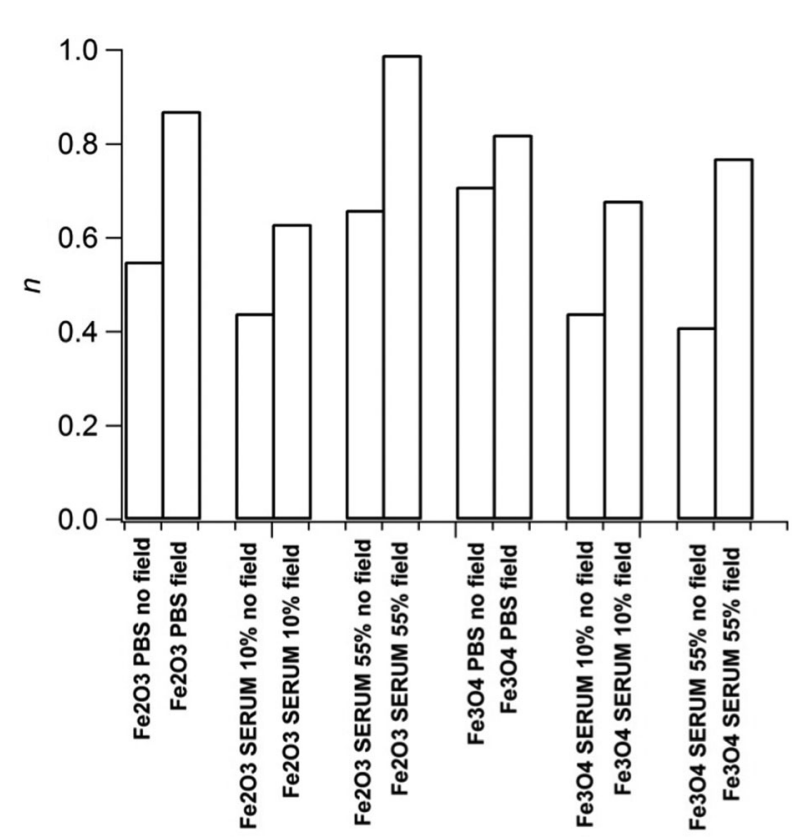

Fig. 6 Schematic graph of the diffusion exponent $n$ extrapolated from the fitting of the kinetic curves with the Ritger-Peppas equation. 
aggregates that hinder the $\mathrm{CF}$ release, resulting in a low response to the LF-AMF.

In terms of the diffusion exponent $n$, the results are summarized in Fig. 6 to make their comparison easier. All the investigated samples display a release kinetic that ranges between the Fickian and the anomalous mechanism.

Furthermore, irrespective of the type of magnetic nanoparticle used and the presence of serum proteins, all the samples display an increase of the $n$ value when the LF-AMF is applied. The results clearly suggest the generation of anomalous release pathways at the level of the ML membrane. In particular, the sample with serum $55 \%$ and $\gamma-\mathrm{Fe}_{2} \mathrm{O}_{3}$ NPs after the LF-AMF treatment displays the highest $n$ value among the investigated samples, indicating a major change in the structure of the ML membrane. These results further highlight the importance of the surface properties of MNPs in the design of remotely triggerable drug delivery vehicles, as well as the crucial role of serum in modulating the LF-AMF effect.

\section{Conclusions}

In this work we report on the preparation, the characterization and the magnetically triggered drug release properties of magnetoliposomes (MLs) prepared either with oleic acid-coated $\gamma-\mathrm{Fe}_{2} \mathrm{O}_{3}$ or citrate-coated $\mathrm{Fe}_{3} \mathrm{O}_{4}$ magnetic nanoparticles (MNPs) and dispersed in biological fluids. The investigation was carried out in order to evaluate how the eventual interaction of phospholipid vesicles and magnetic nanoparticles with biological fluids affects the aggregation and the release properties of MLs, especially when they are subjected to a low frequency alternating magnetic field (LF-AMF).

The co-extrusion of MNPs brings an increase in the average size of liposomes when citrate-coated $\mathrm{Fe}_{3} \mathrm{O}_{4}$ magnetic nanoparticles are used, also accompanied by an increase in the polydispersity in the case of oleic-acid coated $\gamma-\mathrm{Fe}_{2} \mathrm{O}_{3}$ NPs. No major changes are observed in response to the application of the LF-AMF when MNPs are located within the water pool (citrate coated $\mathrm{Fe}_{3} \mathrm{O}_{4}$ NPs), while aggregation between MLs and a consequent increase in the average size are observed when MNPs are located in the proximity of the ML bilayer (oleic acid coated $\gamma-\mathrm{Fe}_{2} \mathrm{O}_{3} \mathrm{NPs}$ ).

The surface functionalization of MNPs and their consequent localization in the ML water pool or at the membrane level are also crucial for the release properties. Hydrophobic MNPs could be uploaded in a much smaller amount with respect to hydrophilic nanoparticles, consistent with the much larger volume available in the water pool than in the proximity of the bilayer. Nevertheless, the effect of oleic-acid coated NPs is significantly stronger than that of citrate-coated NPs. Furthermore, their effect is retained also in the presence of serum proteins.

The comparison between the results obtained in PBS buffer with those in the presence of serum proteins shows a clear decrease in the permeability of the membrane. The typical increase in the amount of released drug when the LF-AMF is applied is strongly dampened as a function of the serum concentration, suggesting a healing effect at the membrane level. Nevertheless, when $\gamma-\mathrm{Fe}_{2} \mathrm{O}_{3}$ NPs are used the magnetic responsivity is retained, while this is nearly lost with citrate coated $\mathrm{Fe}_{3} \mathrm{O}_{4}$ NPs.

Considering the obtained results, the importance of testing the release efficiency of self-assembled drug delivery systems in biological fluids is clear, in order to understand their behaviour in the presence of proteins and biomolecules that can interact with them. In particular, the surface properties of MNPs are crucial for their localization in liposomes either in the water pool or at the membrane level. As a consequence, the effect of the LF-AMF on the drug release properties is strongly different, demonstrating how the hydrophilicity/hydro-phobicity of the MNP surface is a key parameter to tune the membrane permeability.

Here, it still remains unclear if the major destabilization observed after magnetic exposure in the biological fluids was directly bound to the protein adsorption or to a combined effect of a first destabilization of the membrane due to the presence of MNPs, which led to a further extensive agglomeration.

\section{Acknowledgements}

We thank Prof. R. Udisti and Dr F. Rugi for ICP-OES measurements. CSGI is acknowledged for financially supporting this work.

\section{References}

1 T. M. Allen and P. R. Cullis, Science, 2004, 303, 1818-1822.

2 M. C. Woodle, Adv. Drug Delivery Rev., 1995, 16, 249-265.

3 V. P. Torchilin, Nat. Rev. Drug Discovery, 2005, 4, 145-160.

4 G. J. R. Charrois and T. M. Allen, Biochim. Biophys. Acta, 2003, 1609, 102-108.

5 W. T. Al-Jamal, K. T. Al-Jamal, P. H. Bomans, P. M. Frederik and K. Kostarelos, Small, 2008, 4, 1406-1415.

6 S. Nappini, M. Bonini, F. B. Bombelli, F. Pineider, C. Sangregorio, P. Baglioni and B. Nordèn, Soft Matter, 2011, 7, 1025-1037.

7 J. A. Kloepfer, N. Cohen and J. L. Nadeau, J. Phys. Chem. B, 2004, 108, 17042-17049.

8 S. Nappini, M. Bonini, F. Ridi and P. Baglioni, Soft Matter, 2011, 7, 4801-4811.

9 D. Needham and M. W. Dewhirst, Adv. Drug Delivery Rev., 2001, 53, 285-305.

10 J. K. Mills, G. Eichenbaum and D. Needham, J. Liposome Res., 1999, 9, 275-290.

11 P. C. M. Babincová, Bioelectrochemistry, 2002, 55, 17-19.

12 H.-Y. Lin and J. L. Thomas, Langmuir, 2003, 19, 1098-1105.

13 A. Schroeder, Y. Avnir, S. Weisman, Y. Najajreh, A. Gabizon, Y. Talmon, J. Kost and Y. Barenholz, Langmuir, 2007, 23, 4019-4025.

14 S. Lesieur, C. Grabielle-Madelmont, C. Ménager, V. Cabuil, D. Dadhi, P. Pierrot and K. Edwards, J. Am. Chem. Soc., 2003, 125, 5266-5267.

15 S. Nappini, F. B. Bombelli, M. Bonini, B. Nordèn and P. Baglioni, Soft Matter, 2009, 6, 154-162.

16 S. Laurent, D. Forge, M. Port, A. Roch, C. Robic, L. Vander Elst and R. N. Muller, Chem. Rev., 2008, 108, 2064-2110. 
17 S.-H. Hu, T.-Y. Liu, H.-Y. Huang, D.-M. Liu and S.-Y. Chen, Langmuir, 2008, 24, 239-244.

18 N. Kohler, C. Sun, J. Wang and M. Zhang, Langmuir, 2005, 21, 8858-8864.

19 H. S. Ekapop Viroonchatapan, J. Controlled Release, 1997, 46, 263-271.

20 R. E. Rosensweig, J. Magn. Magn. Mater., 2002, 252, 370-374.

21 A. Ito, Y. Kuga, H. Honda, H. Kikkawa, A. Horiuchi, Y. Watanabe and T. Kobayashi, Cancer Lett., 2004, 212, 167-175.

22 G. Beaune, C. Menager and V. Cabuil, J. Phys. Chem. B, 2008, 112, 7424-7429.

23 V. M. De Paoli, S. H. De Paoli Lacerda, L. Spinu, B. Ingber, Z. Rosenzweig and N. Rosenzweig, Langmuir, 2006, 22, 5894-5899.

24 M. D. P. Zonghuan Lu, Langmuir, 2005, 21, 2042-2050.

25 T.-Y. Liu, S.-H. Hu, K.-H. Liu, D.-M. Liu and S.-Y. Chen, J. Controlled Release, 2008, 126, 228-236.

26 M. Bonini, D. Berti and P. Baglioni, Curr. Opin. Colloid Interface Sci., 2013, 18, 459-467.

27 T. Cedervall, I. Lynch, S. Lindman, T. Berggård, E. Thulin, H. Nilsson, K. A. Dawson and S. Linse, Proc. Natl. Acad. Sci. U. S. A., 2007, 104, 2050-2055.

28 M. P. Monopoli, D. Walczyk, A. Campbell, G. Elia, I. Lynch, F. Baldelli Bombelli and K. A. Dawson, J. Am. Chem. Soc., 2011, 133, 2525-2534.

29 D. Walczyk, F. B. Bombelli, M. P. Monopoli, I. Lynch and K. A. Dawson, J. Am. Chem. Soc., 2010, 132, 5761-5768.

30 G. Caracciolo, D. Pozzi, S. Candeloro De Sanctis, A. Laura Capriotti, G. Caruso, R. Samperi and A. Lagana, Appl. Phys. Lett., 2011, 99, 033702.

31 D. E. Owens III and N. A. Peppas, Int. J. Pharm., 2006, 307, 93-102.

32 P. Camner, M. Lundborg, L. Låstbom, P. Gerde, N. Gross and C. Jarstrand, J. Appl. Physiol., 2002, 92, 2608-2616.

33 A. Cifuentes-Rius, H. de Puig, J. C. Y. Kah, S. Borros and K. Hamad-Schifferli, ACS Nano, 2013, 7, 10066-10074.
34 C. A. Monnier, D. Burnand, B. Rothen-Rutishauser, M. Lattuada and A. Petri-Fink, Eur. J. Nanomed., 2014, 6, 201-215.

35 P. Berger, N. B. Adelman, K. J. Beckman, D. J. Campbell, A. B. Ellis and G. C. Lisensky, J. Chem. Educ., 1999, 76, 943. 36 T. Hyeon, Chem. Commun., 2003, 927-934.

37 M.-S. Martina, J.-P. Fortin, C. Ménager, O. Clément, G. Barratt, C. Grabielle-Madelmont, F. Gazeau, V. Cabuil and S. Lesieur, J. Am. Chem. Soc., 2005, 127, 10676-10685.

38 R. Sabaté, R. Barnadas-Rodríguez, J. Callejas-Fernández, R. Hidalgo-Álvarez and J. Estelrich, Int. J. Pharm., 2008, 347, 156-162.

39 I. D. Morrison, E. F. Grabowski and C. A. Herb, Langmuir, 1985, 1, 496-501.

40 M. A. Singh, S. S. Ghosh and R. F. Shannon Jnr, J. Appl. Crystallogr., 1993, 26, 787-794.

41 J. T. SH Chen, Phys. Rev. Lett., 1986, 57, 2583-2586.

42 R. S. Armen, O. D. Uitto and S. E. Feller, Biophys. J., 1998, 75, 734-744.

43 S.-H. C. Michael Kotlarchyk, J. Chem. Phys., 1983, 79, 2461-2469.

44 P. Bartlett and R. H. Ottewill, J. Chem. Phys., 1992, 96, 3306-3318.

45 G. V. Schulz, Z. Phys. Chem., 1939, 25, B43.

46 J. B. Hayter and J. Penfold, Mol. Phys., 1981, 42, 109-118.

47 M. Bonini, E. Fratini and P. Baglioni, Mater. Sci. Eng., C, 2007, 27, 1377-1381.

48 F. Nallet, R. Laversanne and D. Roux, J. Phys. II, 1993, 3, 487-502.

49 S. R. Kline, J. Appl. Crystallogr., 2006, 39, 895-900.

50 G. D. Bothun, J. Nanobiotechnol., 2008, 6, 13.

51 D. Y. Arifin, L. Y. Lee and C.-H. Wang, Adv. Drug Delivery Rev., 2006, 58, 1274-1325.

52 L. Serra, J. Doménech and N. A. Peppas, Biomaterials, 2006, 27, 5440-5451.

53 P. L. Ritger and N. A. Peppas, J. Controlled Release, 1987, 5, 23-36. 